\title{
Effects of two human chorionic gonadotropin doses administered to the ovarian states during the in vitro fertilization and embryo transfer program
}

\author{
MINGXING MA ${ }^{1}$, JIALIN WANG ${ }^{1}$, LIJUN XU ${ }^{1}$, QINXI ZHANG ${ }^{1}$, BOTAO DU ${ }^{1}$, XIAOYING JIANG ${ }^{2}$, QINGLI SHI ${ }^{1}$, \\ LILI ZHOU $^{1}$, BAOXIN LI $^{3}$, HIDEKAZU SAITO ${ }^{3}$ and HIROHISA KURACHI ${ }^{3}$ \\ ${ }^{1}$ Department of Obstetrics and Gynecology, The Second Affiliated Hospital of Harbin Medical University, Harbin, \\ Heilongjiang 150086; ${ }^{2}$ Department of Obstetrics and Gynecology, Harbin Red Cross Hospital, Harbin, Heilongjiang 150076, \\ P.R. China; ${ }^{3}$ Department of Obstetrics and Gynecology, Yamagata University, School of Medicine, Yamagata 990-9585, Japan
}

Received September 18,2014; Accepted October 20, 2014

DOI: 10.3892/br.2014.391

\begin{abstract}
The aim of the present study was to examine the effects of the human chorionic gonadotropin (hCG) dose on the pulsatility indices (PI) of the intraovarian artery on the day of follicle aspiration and the oocyte quality, intrafollicular oxidative stress and luteinization. PI was also measured on the day of hCG administration. A total of 15 patients were undergoing the in vitro fertilization and embryo transfer (IVF-ET) program. To estimate whether there was any difference between the intraovarian artery blood flow and oocyte development of the same patients treated with 5,000 or 10,000 IU hCG, the intraovarian artery blood flow was measured by transvaginal color ultrasonography pulsed wave Doppler, and the follicular fluids and the granulosa cells were collected at follicle aspiration. There were statistically significant differences between the same patients undergoing the two different hCG-dose treatments in which the first protocol included 10,000 IU and the second protocol included 5,000 IU hCG treatment. These differences were apparent in the PI of intraovarian artery blood flow on the day of follicle aspiration $(\mathrm{P}=0.0023)$, in the incidence of apoptosis in cumulus (ApoC) and mural (ApoM) granulosa cells (ApoC, $\mathrm{P}=0.0077$; ApoM, $\mathrm{P}=0.0128)$, in the total oocytes retrieved $(\mathrm{P}=0.0342)$ and in the follicle fluid progesterone concentration $(\mathrm{P}=0.0044)$. There were no significant differences between the two protocols in the PI of intraovarian artery blood flow on the day of hCG administration $(\mathrm{P}=0.4326)$, serum steroid on the day of follicle aspiration [serum $\mathrm{P}, \mathrm{P}>0.9999$; serum estradiol $\left(\mathrm{E}_{2}\right), \mathrm{P}=0.8589$ ],
\end{abstract}

Correspondence to: Dr Botao Du, Department of Obstetrics and Gynecology, The Second Affiliated Hospital of Harbin Medical University, 246 Xuefu Road, Harbin, Heilongjiang 150086, P.R. China

E-mail: botaodu34@hotmail.com

Key words: human chorionic gonadotropin, oocyte maturation, intraovarian artery blood flow, pulsatility index, granulosa cells follicle fluid $\mathrm{E}_{2}$ concentration $(\mathrm{P}=0.8939)$, mature oocyte rate $(\mathrm{P}=0.3743)$ and total mature oocytes retrieved $(\mathrm{P}=0.2026)$. In conclusion, the dose of hCG administration can significantly affect the intraovarian artery blood flow and the development of follicles and oocytes in an IVF-ET program.

\section{Introduction}

Ovulation generates oxidative stress and is therefore comparable to an inflammatory reaction. Similar inflammatory changes initially occur in the theca interna and granulosa layers of follicles in response to chorionic gonadotropin stimulation during the luteinization process. Different cytokines, kinnins, prostaglandins, proteolytic enzymes, nitric oxide and various steroids are produced during the final hours prior to follicle rupture (1). These events are believed to have effects on the blood flow in the ovaries during the periovulatory period.

The midcycle gonadotropin surge is a major factor in the changes of ovulation. Rapidly increasing levels of luteinizing hormone (LH) generate numerous critical changes in oocytes and follicular cells, which further modify the steroid and protein micro- and macroenvironment. These physiological changes have a significant role in oocyte normal maturation, the ovulation process and the subsequent fertilization and implantation (2). Due to the inconsistency of the spontaneous LH surges during controlled ovarian stimulation in any of its forms, the increasing use of gonadotropin-releasing hormone-agonist ( $\mathrm{GnRH}-\mathrm{a})$ is used in all the successful ovarian stimulation programs to affect the eventual triggering of oocyte maturation and ovulation (3).

Due to the specific degree of homology between human chorionic gonadotropin (hCG) and LH, hCG has been used as a surrogate for the LH surge. These two hormones are complex heterodimeric glycoproteins with molecular weights of $>30$ and $40 \mathrm{kDa}$ for recombinant human $\mathrm{LH}$ and hCG, respectively, and also have identical $\alpha$-subunits and a high cystine content. Of note, these two hormones have the same natural function, which is to cause luteinization and support lutein cells (4). Norjavaara et al (5) determined the redistribution of ovarian blood flow following the injection of hCG in the adult pseudopregnant rat and found that there was no hCG-induced change 
in the luteal blood flow on days 2, 6 and 11 of pseudopregnancy. However, in the remaining ovary the blood flow increased $>2$-fold, resulting in redistribution of the blood flow.

Previously, pulsed wave Doppler has also been used to estimate the association between the intraovarian artery blood flow and the growth of ovarian follicles. Nargund et al (6) investigated the association between the ultrasound-derived indices of blood flow in individual follicles at baseline on the day of the administration of hCG and the following recoveries of oocytes or the production of preimplantation embryos. There was a significant correlation between the detection of follicular blood flow and the recovery of an oocyte.

The quality of a follicle and an oocyte has been estimated in terms of the incidence of apoptosis in granulosa cells in the patients involved in in vitro fertilization and embryo transfer (IVF-ET) treatment. Aging and endometriosis have been known to affect the quality of follicular growth and oocytes, which is thought to be due to an increased incidence of apoptosis in granulosa cells (7).

In our previous study (8), which assessed 10,000 IU hCG administration, the intraovarian artery blood flow was increased in association with the decreased mature oocyte rate, and the increased incidence of apoptotic granulosa cells on the day of follicle aspiration indicated that the oocyte had overmatured. Therefore, the present study was designed in order to compare the same patients undergoing two protocols; the first protocol with 10,000 IU and the second with 5,000 IU hCG administration, to investigate whether there were any significant differences between the intraovarian artery blood flow and the development of follicles and oocytes in an IVF-ET program between the two protocols.

\section{Materials and methods}

Patients and follicle-stimulation protocol. The study was approved by the Harbin Medical University Hospital Committee for Research on Human Subjects (Heilongjiang, China). Written informed consent was obtained from all the patients and their clinical information was concealed.

The patients in the IVF group $(n=17)$ had the following causes of infertility: Tubal damage $(n=6)$, male factor $(n=6)$ and unknown $(n=5)$. 'Unknown' means that no cause of infertility could be determined by basal body temperature or serum hormones [LH, follicle-stimulating hormone, prolactin, estradiol $\left(E_{2}\right)$, progesterone $(P)$, testosterone $\left.(T)\right]$ measurements, hysterosalpingography, ultrasonography, laparoscopy or semen analysis.

For all the patients administered a GnRH-a, and also buserelin acetate (Suprecur nasal ${ }^{\circledR}$; Sanofi-Aventis, Tokyo, Japan), human menopausal gonadotropins (hMG; Humegon; Kanebo, Tokyo, Japan) and hCG (Profasi; Serono, Geneva, Switzerland) were also administered. The administration of GnRH-a (600 mg/day) started during the midluteal phase. The administration of hMG started on day 3 of the menstrual cycle. hCG injection was administered when the dominant follicle had a mean diameter $\geq 16 \mathrm{~mm}$ with $10,000 \mathrm{IU}$ in the first protocol with 10,000 IU and 5,000 IU hCG administration in the second protocol. As two patients were diagnosed as pregnant in the first protocol, they were excluded from the second protocol.
Measurement of ovarian artery blood flow and follicle aspiration. On the hCG administration day and the follicle aspiration day, both sides of intraovarian artery flow for each patient were measured by transvaginal color ultrasonographic pulsed wave Doppler. The values of pulsatility indices (PI) were printed on a wave-form image. The PI of each patient was defined as the mean value of the two measured sides.

Oocyte retrieval was performed $35 \mathrm{~h}$ after the $\mathrm{hCG}$ administration by follicle aspiration under transvaginal ultrasonographic guidance. All the follicles with a mean diameter $>12 \mathrm{~mm}$ were aspirated, using an 18-gauge needle connected to a connecting tube and $20-\mathrm{ml}$ syringe for suction.

Granulosa cell treatment. The oocyte-cumulus complex was removed from the follicular fluid, washed twice in culture medium (human tubal fluid) and placed in fresh culture medium. The cumulus granulosa cells (ApoC) were mechanically separated from the oocyte using 26-gauge needles under the microscope and placed on glass slides. The mural cell mass was collected from the follicular fluid and washed twice in the culture medium. The ApoC and mural granulosa cells (ApoM) were dispersed separately by hyaluronidase $(0.1 \%$ w/v) and fixed with $4 \%$ neutral formalin on glass slides. The granulosa cells were fixed $<15$ min after the oocyte recovery. Subsequent to drying the glass slides, they were rinsed twice by phosphate-buffered saline. The fixed granulosa cells were stained with Hoechst 33258 fluorescent dye solution $(0.5 \mathrm{mg} / \mathrm{ml}$ in distilled water; Wako, Osaka, Japan) that contained 1,4-diazabicyclo-2,2,2-octane (Sigma, St. Louis, MO, USA) to prevent fading, and were then mounted with a coverglass.

A total of 1,000 granulosa cells were observed under a fluorescent microscope and the number of apoptotic cells was counted. Apoptotic cells were defined as those with fragmented and uniformly dense nuclei or fragmented cytoplasm containing a uniformly dense fragmented nucleus.

Follicular fluids collection and steroids assay. Each follicular fluid sample was centrifuged at $300 \mathrm{x}$ g for $10 \mathrm{~min}$ and the cleared fluid was stored at $-20^{\circ} \mathrm{C}$ until the assay for $\mathrm{E}_{2}$, $\mathrm{P}$ and $\mathrm{T}$. The quantification of the hormonal concentration was performed using commercially available immunoassays: $\mathrm{E}_{2}$ by time-resolved fluoroimmunoassay (DELFIA Estradiol; Pharmacia, Tokyo, Japan), P by radioimmunoassay (RIA) (DPC progesterone; DPC, Inc., Tokyo, Japan) and free T by RIA (DPC progesterone kit and DPC free testosterone kit; DPC, Inc.). Reliability criterions for all the assays were established. The hormones were assayed in duplicate within the same assay (8).

\section{Assessment of oocyte}

Oocyte quality. Oocyte quality was scored by the incidence of apoptotic granulosa cells with condensed and fragmented nuclei.

Oocyte maturity. Oocyte maturity was judged by the condition of the corona radiata and the ApoC, as described previously. Oocyte-corona-cumulus complexes with a large and loose cumulus and distinct corona radiata were defined as mature, and complexes with a small and dense cumulus and opaque corona radiata were defined as immature (9).

Statistical analysis. The significance of the differences in the data was analyzed by the Student's t-test (two group t-test, 


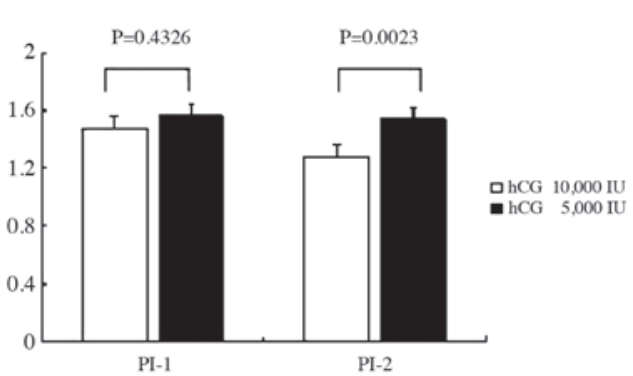

Figure 1. Dose of human chorionic gonadotropin (hCG) affects the intraovarian artery blood flow. There was a significant difference between the two protocols in pulsatility indices (PI) on the day of follicle aspiration (PI-2; $\mathrm{P}=0.0023$ ) but not on the hCG applied day (prior to hCG administration) $(\mathrm{PI}-1 ; \mathrm{P}=0.4326)$. There was no significant difference in the intraovarian artery blood flow between the two protocols on the hCG applied day, but there was a significant decrease in the intraovarian artery blood flow in hCG for 5,000 IU compared to $10,000 \mathrm{IU}$ on the follicle aspiration day.

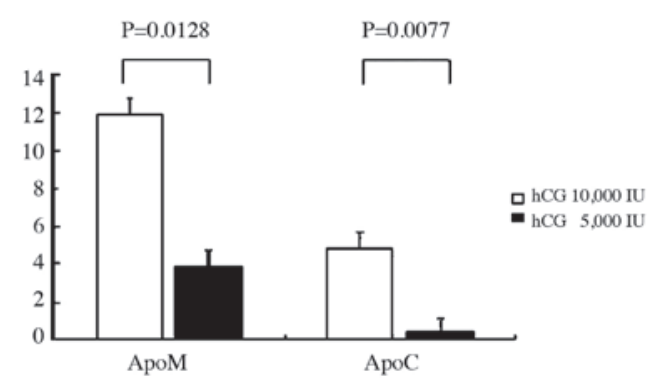

Figure 2. Dose of human chorionic gonadotropin (hCG) affects the maturation of follicles. The 5,000 IU hCG administration significantly decreased the incidence of apoptotic bodies in mural (ApoM; $\mathrm{P}=0.0128$ ) and cumulus (ApoC; $\mathrm{P}=0.0077$ ) granulose cells compared to $10,000 \mathrm{IU}$ hCG administration. This means that $10,000 \mathrm{IU}$ decreased the quality of retrieved oocytes compared to the administration of 5,000 IU hCG.

paired) and Wilcoxon signed-ranks test in computer-assisted statistical analysis. Statistical power was determined by analysis based on sample size and was sufficient to detect the mean differences of approximately one standard deviation. $\mathrm{P}<0.05$ was considered to indicate a statistically significant difference.

\section{Results}

Intraovarian artery blood flow. The difference of intraovarian artery blood flow between the first protocol with 10,000 IU and the second protocol with 5,000 IU hCG administration is shown in Fig. 1. There were no statistically significant differences between the two protocols prior to $\mathrm{hCG}$ administration $(\mathrm{P}=0.4326)$. However, there were statistically significant differences between the two protocols for 10,000 and 5,000 IU hCG administration in the follocle aspiration day $(\mathrm{P}=0.0023)$, which indicated that the nitraovarian artery blood flow significantly decreased due to 5,000 compared to $10,000 \mathrm{IU}$ hCG administration.

Incidence of apoptosis. The incidence difference of apoptotic granulosa cell between the two protocols is shown in Fig. 2. There were statistically significant differences between the two protocols for 10,000 and 5,000 IU hCG administration in the incidence of ApoC $(\mathrm{P}=0.0077)$ and ApoM $(\mathrm{P}=0.0128)$.
Table I. Difference between the two protocols for the concentration of steroid hormone.

\begin{tabular}{|c|c|c|c|}
\hline \multirow[b]{2}{*}{ Steroid hormone } & \multicolumn{2}{|c|}{ hCG } & \multirow[b]{2}{*}{ P-value } \\
\hline & $10,000 \mathrm{IU}$ & $5,000 \mathrm{IU}$ & \\
\hline Follicular estradiol & $460.6 \pm 152.2$ & $529.6 \pm 454.4$ & 0.8393 \\
\hline $\begin{array}{l}\text { Follicular } \\
\text { progesterone }\end{array}$ & $5.9 \pm 1.9$ & $2.3 \pm 3.3$ & $0.0044^{\mathrm{a}}$ \\
\hline $\begin{array}{l}\text { Serum estradiol } \\
\text { aspiration day }\end{array}$ & $865.0 \pm 573.9$ & $815.6 \pm 535.2$ & 0.8589 \\
\hline $\begin{array}{l}\text { Serum progesterone } \\
\text { aspiration day }\end{array}$ & $5.0 \pm 3.9$ & $4.8 \pm 2.5$ & 0.9999 \\
\hline $\begin{array}{l}\text { Serum estradiol } \\
\text { hCG applied day }\end{array}$ & $1175.2 \pm 951.0$ & $839.3 \pm 516.8$ & 0.3078 \\
\hline $\begin{array}{l}\text { Serum progesterone } \\
\text { hCG applied day }\end{array}$ & $0.7 \pm 0.4$ & $1.6 \pm 1.2$ & 0.0546 \\
\hline
\end{tabular}

${ }^{\mathrm{a}} \mathrm{P}<0.05$. Administration of $10,000 \mathrm{IU}$ significantly increased the concentration of follicular progesterone compared to 5,000 IU administration ( $\mathrm{P}=0.0044$ ), but not follicular estradiol. No significant difference was found between the two protocols for serum estradiol and progesterone on $\mathrm{hCG}$ applied day (estradiol, $\mathrm{P}=0.3078$; progesterone, $\mathrm{P}=0.0546$ ) or follicle aspiration day (estradiol, $\mathrm{P}=0.8589$; progesterone, $\mathrm{P}=0.9999$ ). hCG, human chorionic gonadotropin.

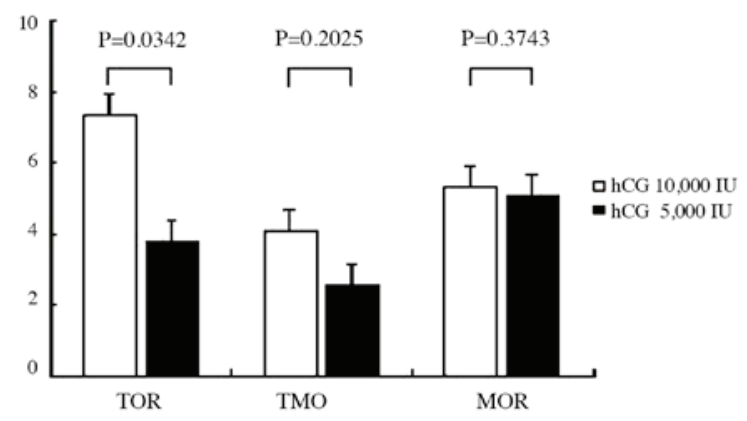

Figure 3. Effect of the human chorionic gonadotropin (hCG) dose on retrieved oocytes. The 10,000 IU hCG administration protocol significantly increased the total oocytes retrieved (TOR; $\mathrm{P}=0.0342$ ) compared to 5,000 IU. However, no difference was found between the two protocols in the total mature oocytes (TMO) or the rate of mature oocytes retrieved (MOR).

This indicated that there was an increased incidence of ApoC and ApoM in 10,000 compared to in 5,000 IU administration.

Differences in hCG administration. The differences between the two protocols in the follicle fluid progesterone and estradiol concentration are shown in Table I. There was a statistically significant difference between the two protocols for 10,000 and $5,000 \mathrm{IU}$ hCG administration in the follicle fluid progesterone concentration ( $\mathrm{P}=0.0044)$, as $10,000 \mathrm{IU}$ significantly increased the follicle fluid progesterone concentration compared to $5,000 \mathrm{IU}$ administration. There was no statistically significant difference between the two protocols in the follicle fluid estradiol concentration $(\mathrm{P}=0.8939)$. There were also no differences between the two protocols in serum progesterone and estradiol concentration ( $\left.\mathrm{P}, \mathrm{P}>0.9999 ; \mathrm{E}_{2}, \mathrm{P}=0.8589\right)$.

The differences between the two protocols in total oocytes retrieved, total mature oocytes retrieved and mature oocyte rate are shown in Fig. 3. There were statistically significant 
differences between the two protocols in total oocytes retrieved $(\mathrm{P}=0.0342)$, as $10,000 \mathrm{IU}$ significantly increased the total oocytes retrieved compared to 5,000 IU administration. There were no statistically significant differences between the two protocols in total mature oocytes retrieved $(\mathrm{P}=0.2026)$ and mature oocyte rate $(\mathrm{P}=0.3743)$.

\section{Discussion}

Currently in the IVF-ET program, hCG is used in all successful ovarian stimulation programs to affect the eventual triggering of oocyte maturation and ovulation and it has been used as a surrogate LH surge due to the degree of homology between the two hormones. hCG has a slower plasma metabolic clearance, consisting of a rapid phase in the initial 5-9 $\mathrm{h}$ and a slower phase in the 1-1.3 days after hCG administration. After $36 \mathrm{~h}$, the calculated half-life of hCG was 2-3.2 days (10).

The study by Bjercke et al (11) determined whether there was any difference in the outcome of IVF when retrieval of oocytes was performed 34 (group A) and $38 \mathrm{~h}$ (group B) after hCG injection in a total 170 patients with tubal failure. The study found no significant difference for any of the parameters tested for in groups A and B. Nargund et al (12) investigated whether the hCG-oocyte collection interval had an influence on the oocyte recovery rate, fertilization rate and outcome of IVF-ET cycles with 533 consecutive patients undergoing their first IVF-ET cycle. There was no significant difference found among the hCG-oocyte collection intervals examined (33-41 h). None of the females studied had ovulated prior to oocyte collection.

Thus, we hypothesized that the oocyte collection intervals within 33-41 h cannot be significantly affected after the same dose of hCG administration. Furthermore, in the present study, follicle aspiration was attempted at $36 \mathrm{~h}$ after hCG administration. Therefore, the effect of oocyte collection intervals following hCG administration can be accepted.

In the study, the parameters between the two protocols with the same group of patients following 10,000 and 5,000 IU hCG administration were analyzed by Student's t-test (two group t-test, paired) and Wilcoxon signed-ranks test in computer-assisted statistical analysis. There were statistically significant differences between the two protocols in the intraovarian artery blood flow, the incidence of ApoC and ApoM, follicle fluid progesterone concentration and total oocytes retrieved.

There were numerous types of regional hormones, enzymes and cytokines that are correlated with a similar inflammatory reaction of the intravessels in the ovulation process. Certain substances can promote the increase of dilated vessels and extravasation of resin from weakened vessels, promote the production of progesterone following a hCG surge, promote the oocyte maturation, regulate the activity of collagenase and prolylhydroxylase for follicle rupture and prevent the premature degeneration of intrafollicular oocytes $(1,13)$. Expecting the effect of hCG-oocyte retrieval intervals following hCG administration, it can be concluded that the differences between the two protocols with 10,000 and 5,000 IU hCG administration was due to the dose of hCG administration and not intervals. The 5,000 IU hCG administration protocol significantly increased PI and decreased follicle fluid P and total oocytes retrieved compared to the 10,000 IU hCG administration protocol, due to the lower hCG dose. However, the decreased ApoM and ApoC can be explained by the presence of more oocytes in the premature step in the 5,000 IU compared to the 10,000 IU hCG protocol. Therefore, it was more difficult for oocyte retrieval by follicle aspiration and as a result the total oocytes retrieved were significantly decreased in comparison. The serum was recovered 35-36 $\mathrm{h}$ after hCG administration, not 5-9 $\mathrm{h}$, so there were no siginificant deference between the two protocols in serum progesterone and estradiol concentration.

In conclusion, the present study demonstrates that there were statistically significant differences between the two protocol with 10,000 and 5,000 IU hCG administration in the intraovarian artery blood flow, incidence of apoptotic granulosa cells, follicle fluid progesterone concentration and total oocyte retrieved in the IVF-ET program. The dose of hCG administration can affect the oocyte maturation or the outcome of the IVF-ET program.

\section{Acknowledgements}

The present study was supported in part by the Education Overseas Scholars (grant no. 1151hq041) of Heilongjiang Province Education Administration and the Provincial Health Issues (major project, grant no. 2006-098) of Heilongjiang Province Health Bureau.

\section{References}

1. Espey LL: Current status of the hypothesis that mammalian ovulation is comparable to an inflammatory reaction. Biol Reprod 50: 233-238, 1994.

2. Shoham Z, Schacter M, Loumaye E, Weissman A, MacNamee M and Insler V: The luteinizing hormone surge - the final stage in ovulation induction: modern aspects of ovulation triggering. Fertil Steril 64: 237-251, 1995.

3. European Recombinant LH Study Group: Human recombinant luteinizing hormone is as effective as, but safer than, urinary human chorionic gonadotropin in inducing final follicular maturation and ovulation in in vitro fertilization procedures: results of a multicenter double-blind study. J Clin Endocrinol Metab 86: 2607-2618, 2001.

4. Weissman A, Lurie S, Zalel Y, Goldchmit R and Shoham Z: Human chorionic gonadotropin: pharmacokinetics of subcutaneous administration. Gynecol Endocrinol 10: 273-276, 1996.

5. Norjavaara E, Olofsson J, Gåfvels $M$ and Selstam G: Redistribution of ovarian blood flow after injection of human chorionic gonadotropin and luteinizing hormone in the adult pseudopregnant rat. Endocrinology 120: 107-114, 1987.

6. Nargund G, Doyle PE, Bourne TH, Parsons JH, Cheng WC, Campbell S and Collins WP: Ultrasound derived indices of follicular blood flow before HCG administration and the prediction of oocyte recovery and preimplantation embryo quality. Hum Reprod 11: 2512-2517, 1996.

7. Nakahara K, Saito H, Saito T, Ito M, Ohta N, Takahashi T and Hiroi M: The incidence of apoptotic bodies in membrana granulosa can predict prognosis of ova from patients participating in in vitro fertilization programs. Fertil Steril 68: 312-317, 1997.

8. Du B, Takahashi K, Ishida GM, Nakahara K, Saito H and Kurachi H: Usefulness of intraovarian artery pulsatility and resistance indices measurement on the day of follicle aspiration for the assessment of oocyte quality. Fertil Steril 85: 366-370, 2006.

9. Saito H, Saito T, Kaneko T, Sasagawa I, Kuramoto T and Hiroi M: Relatively poor oocyte quality is an indication for intracy toplasmic sperm injection. Fertil Steril 73: 465-469, 2000.

10. Damewood MD, Shen W, Zacur HA, Schlaff WD, Rock JA and Wallach EE: Disappearance of exogenously administered human chorionic gonadotropin. Fertil Steril 52: 398-400, 1989.

11. Bjercke S, Tanbo T, Dale PO and Abyholm T: Comparison between two hCG-to-oocyte aspiration intervals on the outcome of in vitro fertilization. J Assist Reprod Genet 17: 319-322, 2000. 
12. Nargund G, Reid F and Parsons J: Human chorionic gonadotropin-to-oocyte collection interval in a superovulation IVF program. A prospective study. J Assit Reprod Genet 18: 87-90, 2001.
13. Wang LJ and Norman RJ: Concentrations of immunoreactive interleukin-1 and interleukin-2 in human preovulatory follicular fluid. Hum Reprod 7: 147-150, 1992. 